\title{
Compact Operators on Hilbert Spaces
}

\author{
Sara Nozari \\ Department of Mathematics, Farsan Branch, Islamic Azad University, Farsan, Iran \\ Email: Email math@ymail.com
}

Received 24 June 2014; revised 4 August 2014; accepted 14 September 2014

Copyright (C) 2014 by author and OALib.

This work is licensed under the Creative Commons Attribution International License (CC BY).

http://creativecommons.org/licenses/by/4.0/

CC) $\underset{\mathrm{EY}}{\mathrm{C}}$ Open Access

\begin{abstract}
In this paper, we obtain some results on compact operators. More specially, we prove that if $T$ is a unitary operator on a Hilbert space $H$, then it is compact if and only if $H$ has a finite dimension. Also, we prove that, if $H$ is a Hilbert space with Heine-Borel property, then $K(H)=B L(H)$.
\end{abstract}

\section{Keywords}

\author{
Compact Operator, Linear Projections, Heine-Borel Property
}

Subject Areas: Mathematical Analysis, Quantum Mechanics

\section{Introduction}

Surely, the operator theory is the heart of functional analysis. This means that if one wishes to work on functional analysis, he/she must study the operator theory. In operator theory, we study operators and connection between it and other mathematical subjects. Recently, many mathematicians have studied compact operators; See [1]-[3] for more details. One of the important applications of these operators is solving the integral equations. In this paper, we obtain some results on compact operators. First of all we recall some definitions and results that are needed in the sequel. Any unexplained notion and definitions can be found in [4].

Definition 1.1: Let $T$ be a linear operator on a Hilbert space $H . T$ is called to be compact if for every bounded subset $M$ of $H, T(M)$ is compact.

Notation 1.2: The set of all compact operators on $H$ is denoted by $K(H)$. It is easy to see that $K(H)$ is a linear subspace of $\mathrm{BL}(H)$.

Lemma 1.3: Let $H$ be an infinite dimensional Hilbert space. Then the identity operator on $H$ is not compact.

Theorem 1.4: Any bounded operator with finite rank is compact.

Definition 1.5: An operator $P$ on a normed space $X$ is called a projection if $P^{2}=P$.

Proposition 1.6: Let $P$ be a linear projection on $H$. Then $P$ is compact if and only if it has a finite rank.

Definition 1.7 [5]: We say that a topological vector space $X$ has property Heine-Borel when every bounded and closed subset of $X$ is compact. 


\section{Compact Operators on Hilbert Spaces}

We begin the section with the following theorem.

Theorem 2.1: Let $H$ be an infinite-dimensional Hilbert space and $T$ a compact operator on $H$. Then $T$ is not invertible.

Proof: Let $T$ be invertible. It is well-known and easy to show that the composition of two operator is compact, and at least one of them is compact ([4], th.11.5). Therefore $T T^{-1}=I$ is compact which contradicts to Lemma 1.3.

Corollary 2.2: If $T$ is an invertible operator on an infinite dimensional Hilbert space, it is not compact.

Corollary 2.3: Let $T$ be a bounded operator with a finite rank on an infinite-dimensional Hilbert space $H$. Then $T$ is not invertible.

Proof: By Theorem 1.4, $T$ is compact. Now the proof is completed by Theorem 2.1.

Corollary 2.4: Let $P$ be a linear projection on $H$ with a finite rank. Then it is not invertible.

Proof: Use the Proposition 1.6 together with Theorem 2.1. $\square$

Recall that a self-adjoint operator is an operator which equals to its adjoint.

Theorem 2.5: Let $H$ be an infinite-dimensional Hilbert space and $T$ a compact and self-adjoint operator on $H$. Then $T^{n}$ is not invertible, for each $n \in \mathbb{N}$.

Proof: First note that $T^{*}$ is compact. On the other hand, theorem implies that $T T^{*}$ is compact ([4], Th.9.2 (ii)). Now, since $T$ is self-adjoint, $T^{2}$ is compact and by Theorem 2.1 it is not invertible. In a similar manner, we conclude that $T^{n}$ is not invertible, for each $n \in \mathbb{N}$.

Here, we recall that an operator $T$ is called unitary when $T^{*} T=T T^{*}=I$.

Theorem 2.6: Let $T$ be an unitary operator on $H$. Then $T$ is compact if and only if $H$ has a finite dimension.

Proof: $(\Rightarrow)$ We have $T^{*} T=T T^{*}=I$. Since $T$ is compact, $I$ is compact. Therefore $H$ has a finite dimension (Lemma 1.3).

$(\Leftarrow)$ Since the identity operator $I$ on $H$ is compact (Lemma 1.3), $T^{*} T$ (and also $T T^{*}$ ) is compact. On the other hand, $T T^{*} T=T$, which implies $T$ is compact.

Theorem 2.7: Let $H$ be a real or complex finite dimensional Hilbert space. Then

$$
\operatorname{card}(K(H)) \geq c
$$

where $c$ is the cardinal of continuum.

Proof: Define

$$
A=\{\lambda I: \lambda \in \mathbb{R} \text { or } \mathbb{C}\}
$$

Note that

$$
\operatorname{card}(\mathbb{R} \text { or } \mathbb{C})=c
$$

Hence

$$
\operatorname{card}(A)=c .
$$

$H$ has finite dimension and so $A \subset K(H)$. It follows that

$$
\operatorname{card}(K(H)) \geq c
$$

Thus the proof is finished. $\square$

Remark 2.8: If $H$ is a finite dimensional Hilbert space, any bounded linear operator on $H$ is compact. Although, in general, a bounded linear operator on a Hilbert space $H$ needs not to be compact, in the following we give a sufficient condition for a bounded linear operator to be compact.

Theorem 2.9: Let $H$ be a Hilbert space with Heine-Borel property. Then $K(H)=\operatorname{BL}(H)$.

Proof: It is sufficient to show that $\mathrm{BL}(H) \subset K(H)$. For this, let $T \in \mathrm{BL}(H)$. Then since $H$ has the HeineBorel property, it is easy to show that $T(M)$ is relatively compact, for every bounded set $M$ in $X$, as required.

Corollary 2.10: Let $H$ be a real or complex Hilbert space with Heine-Borel property. Then

$$
\operatorname{card}(K(H)) \geq c
$$


where $c$ is the cardinal of continuum.

Proof: This immediately follows the fact that the cardinal of the set

$$
A=\{\lambda I: \lambda \in \mathbb{R} \text { or } \mathbb{C}\}
$$

is equal to $c . \square$

Corollary 2.11: There is no infinite dimensional Hilbert space with property Heine-Borel.

Proof: By contrary, let $H$ be an infinite dimensional Hilbert space with property Heine-Borel.

Then the identity operator on $H$ is compact (Theorem 2.9) which contradicts to Lemma 1.3. $\square$

Now we reach at the important following result.

Corollary 2.12: A Hilbert space has property Heine-Borel if and only if it has a finite dimension.

Proof: Use Corollary 2.11 together with the well-known fact that finite dimensional spaces have property Heine-Borel.

\section{References}

[1] Spurny, J. (2007) A Note on Compact Operators on Normed Linear Spaces. Expositiones Mathematicae, 25, $261-263$. http://dx.doi.org/10.1016/j.exmath.2006.11.002

[2] De la Sen, M. (2013) On a Class of Self-Adjoint Compact Operators in Hilbert Spaces and Their Relations with Their Finite-Range Truncations. Abstract and Applied Analysis, 2013, 1-15.

[3] Baker, J.M. (1979) A Note On Compact Operators Which Attain Their Norm. Pacific Journal of Mathematics, 82, 319-325. http://dx.doi.org/10.2140/pjm.1979.82.319

[4] Thamban Nair, M. (2002) Functional Analysis-A First Course. Prentice-Hall, New Delhi.

[5] Rudin, W. (1973) Functional Analysis. McGraw-Hill, New York. 